\title{
Brief freezing steps lead to robust immunofluorescence in the Drosophila nervous system
}

Ada Thapa', Shea M Sullivan', Minh Q Nguyen'1, Dominic Buckley', Vy T Ngo', Austin O Dada', Essence Blankinship', Veronica Cloud' \& Ryan D Mohan*,1

\section{ABSTRACT}

Drosophila melanogaster possesses a complex nervous system, regulating sophisticated behavioral outputs, that serves as a powerful model for dissecting molecular mechanisms underlying neuronal function and neurodegenerative disease. Immunofluorescence techniques provide a way to visualize the spatiotemporal organization of these networks, permitting observation of their development, functional location, remodeling and, eventually, degradation. However, basic immunostaining techniques do not always result in efficient antibody penetration through the brain, and supplemental techniques to enhance permeability can compromise structural integrity, altering spatial organization. Here, slow freezing of brains is shown to facilitate antibody permeability without loss of antibody specificity or brain integrity. To demonstrate the advantages of this freezing technique, the results of two commonly used permeation methods - detergentbased and partial proteolytic digestion - are compared.

\section{METHOD SUMMARY}

A brief, slow-freezing step is added to immunofluorescence procedures in order to improve antibody penetration while maintaining physiological sample structure.

\section{KEYWORDS}

antibody $\cdot$ brain $\cdot$ circadian $\cdot$ Drosophila - Drosophila melanogaster • flies • fluorescence $\cdot$ freezing $\cdot \mathrm{IF} \cdot$ imaging $\cdot$ immunofluorescence $\cdot$ microscopy $\cdot$ nervous system - neural circuit - Per - Period - phalloidin • sleep • staining • wake

'University of Missouri - Kansas City, School of Biological \& Chemical Sciences, 5009 Rockhill Rd, Kansas City, MO 64110, USA; *Author for correspondence:MohanRD@umkc.edu

BioTechniques 67: 299-305 (December 2019) 10.2144/btn-2018-0067
Drosophila melanogaster is a powerful model organism for understanding nervous system function. With low molecular redundancy and a moderate number of neurons commanding complex behaviors, the pace of discovery in Drosophila is relatively quick. Regulatory mechanisms discovered in Drosophila consistently provide a roadmap for deciphering conserved pathways in humans [1]. Additionally, a number of neurodegenerative diseases are recapitulated in Drosophila, allowing investigation into mechanisms contributing to these devastating diseases.

A routine method employed to investigate nervous system function is immunofluorescence (IF), because it allows visualization of specific proteins and the structures they comprise, from subcellular to entire networks of related cells. Various approaches to IF exist [2-4]; however, efficient permeation of antibodies through the brain can present a problem in producing consistent, clear images of target proteins and the structures within the brain that bear them. Permeation using proteolytic enzymes such as collagenase [5] can produce more robust staining, but brain morphology is collaterally disrupted, hindering analysis of spatial organization of brain circuits. Additionally, proteases can be costly and other techniques can require day's long antibody incubation [3].

In optimizing IF approaches to examine Drosophila brains, we found that freezing brains in blocking buffer led to more consistent staining of target proteins and increased observation of immunoreactive structures. Importantly, by using freezing methods, brain morphology was preserved in larvae. Additionally, freezing time was relatively short, and expensive proteases were not required. Therefore, freezing techniques are useful alternatives to established immunostaining procedures because they allow for clear, reproducible visualization of target proteins while avoiding major alterations of brain tissue that occur in harsher permeation methods. Time and money are also saved with simple freezing steps.

To demonstrate the effectiveness of the freezing techniques in immunostaining Drosophila brains, results were compared with those of two other techniques: 'basic' and 'collagenase'. In order to show effectiveness at detecting a deeply embedded neural circuit, the Per protein was selected as an antibody target. Per is a well-characterized neuronal pacemaker that is specifically produced in clock neurons [6]. F-actin (labeled with phalloidin) was selected to demonstrate staining of large neuronal structures, and nuclei (visualized with the DNA-binding agent 4',6-diamidino2-phenylindole [DAPI]) were labeled to show maintenance of brain morphology.

Oregon- $R$ (wild-type) third instar larval brains (BDSC Cat \# 5, RRID:BDSC_5) were dissected in $1 \times$ PBS, transferred to $1.5-\mathrm{ml}$ centrifuge tubes containing $4 \%$ formaldehyde, and incubated for $1 \mathrm{~h}$ at room temperature (RT). For the collagenase technique, brains were dissected into cold $1 \times$ PBS, incubated in $1 \times$ collagenase for $5 \mathrm{~min}$ at RT, and washed three times in $1 \times$ PBS by inverting the tube prior to fixation. Whole adult flies (maximum 8 days old, minimum 5 days old) were placed in $4 \%$ formaldehyde plus $0.1 \%$ Tween- 20 and placed at $4{ }^{\circ} \mathrm{C}$ for $4 \mathrm{~h}$ and then dissected into cold $1 \times$ PBS. After fixation, brains were washed three times in $1 \times$ PBS by inverting the tube, then washed three times in $0.5 \%$ PBS Triton X-100 (PBT) by inverting the tube. Afterwards, brains were incubated in PBT for 20 min at RT with nutating. After removing the PBT from the tube, 
Figure 1. Comparison of four immunofluorescence techniques reveals quantitative and qualitative differences in detection of circadian clock neurons. Oregon-R third instar larval brains were dissected and stained using the indicated immunofluorescence techniques. Anti-Per was used to specifically detect circadian clock neurons. Maximum projections from three representative brains are shown from each technique. Scale bars: $20 \mu \mathrm{m}$. Microscope settings were kept constant across techniques. Voxels for each lobe were quantified and the data is shown. A t-test was used to determine whether total signal detected by each technique was significantly different from the basic protocol. Diagrammed are locations of per/tim-expressing cells and their projections in the larval brain, reproduced from Helfrich-Forster [9]

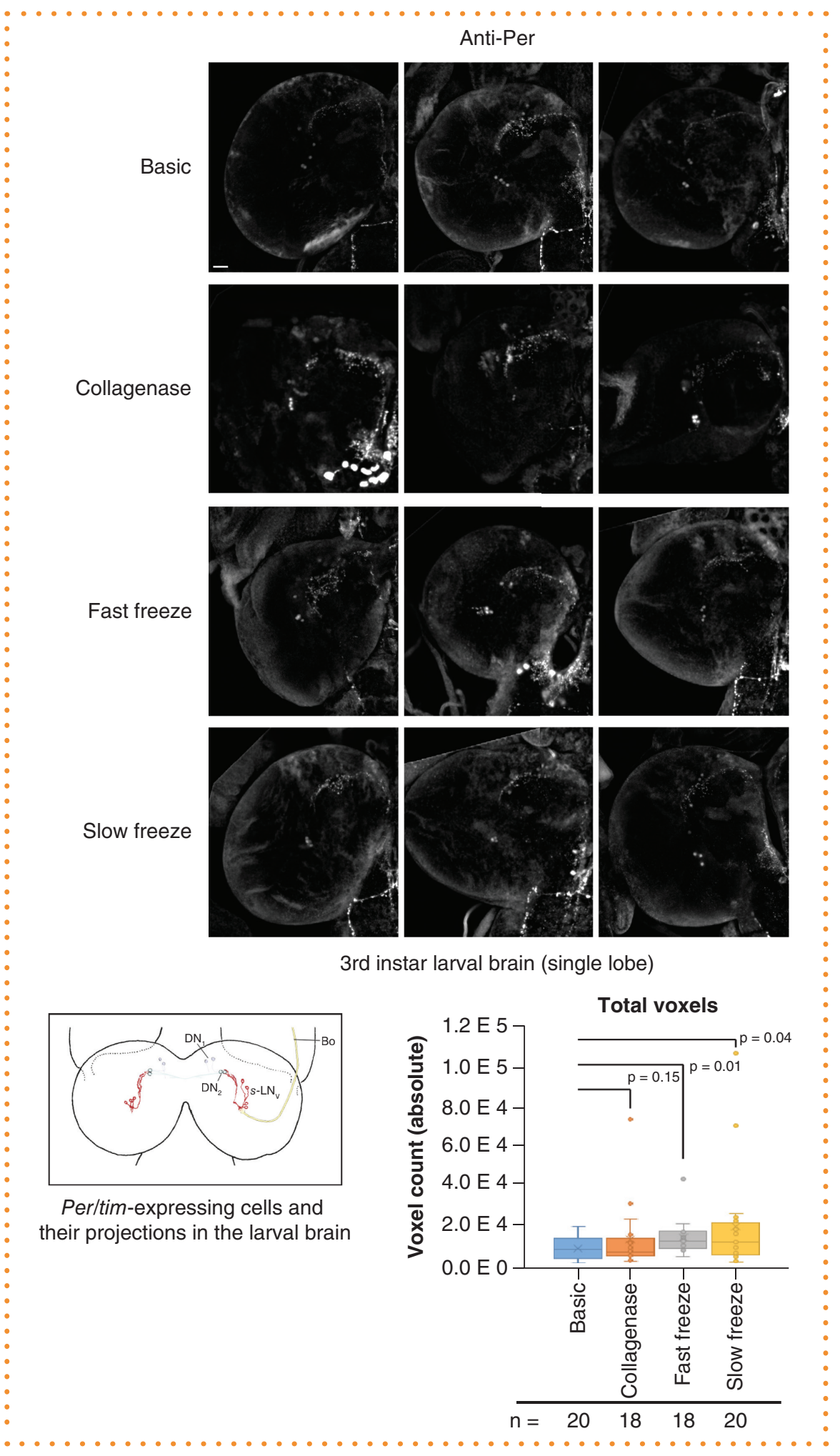

the dry ice with $70 \%$ ethanol for $10 \mathrm{~s}$ for the fast freeze technique. Afterward, brains were thawed/blocked or simply blocked in blocking buffer for $1 \mathrm{~h}$ at RT with nutating. Primary anti-Per antibody [7] was diluted in blocking buffer to a final antibody dilution of 1:10,000. Blocking buffer was removed from the brains and $500 \mu$ l of primary antibody solution was added. Brains were incubated in primary antibody at $4^{\circ} \mathrm{C}$ overnight with nutating. Afterward, brains were washed three times in PBT by inversion, followed by four 10-min washes with nutating using PBT. Secondary anti- 


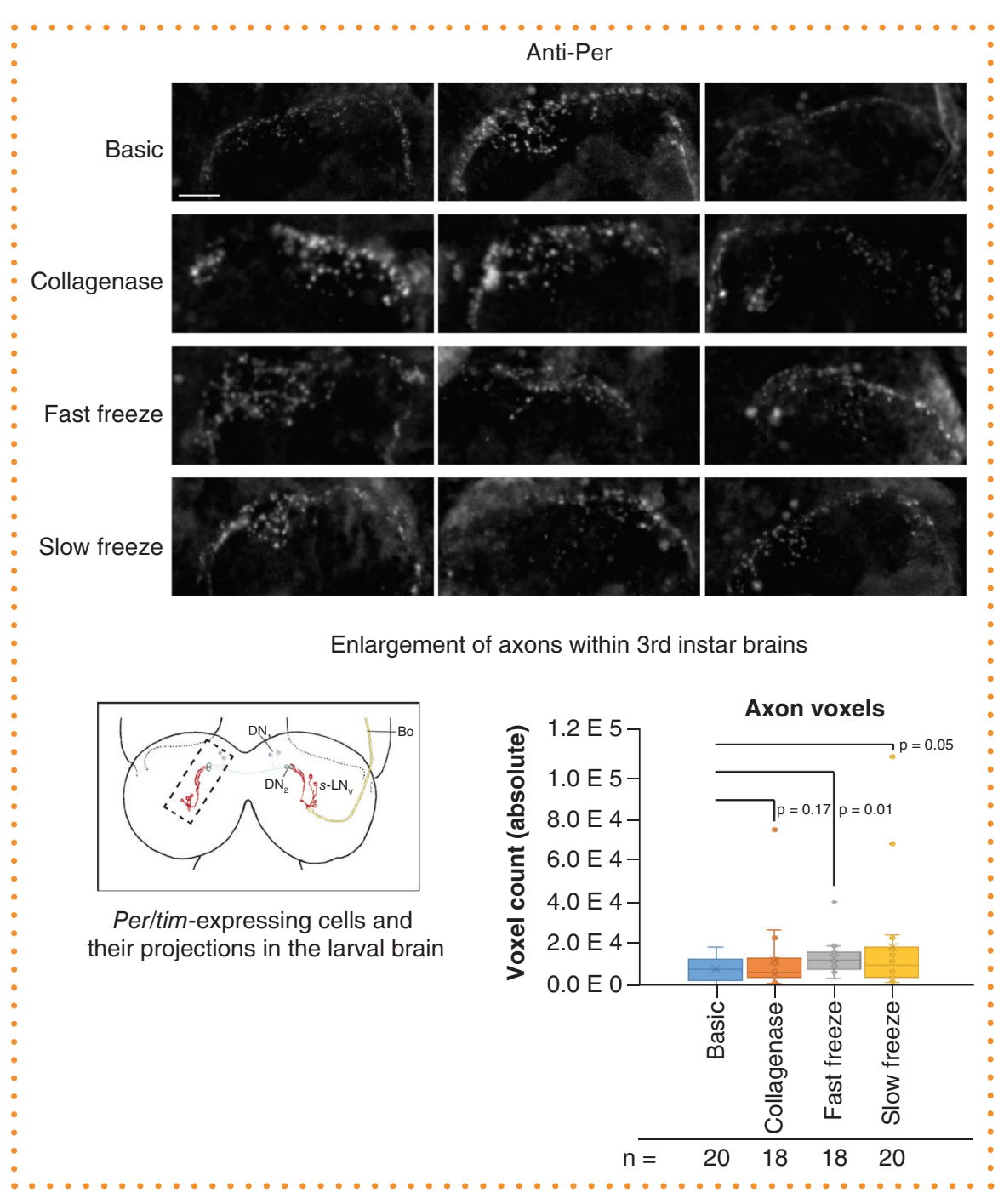

rabbit antibody conjugated to a $488 \mathrm{~nm}$ fluorophore (Thermo Fisher Scientific Cat\# A-11034, RRID:AB_2534095) and phalloidin labeled with a $568 \mathrm{~nm}$ fluorophore (Thermo Fisher Scientific Cat\# A12380, RRID:AB_2315147) were diluted in blocking buffer at a 1:1000 and 1:20 dilution, respectively. PBT was removed from the tube and $200 \mu$ l of secondary/ phalloidin solution was added. Brains were incubated in secondary/phalloidin solution for $4 \mathrm{~h}$ at RT with nutating. Afterward, brains were again washed three times in PBT by inversion and then washed four times for $10 \mathrm{~min}$ at RT with nutating using PBT. After removing the PBT, $50 \mu$ l of mounting solution containing DAPI (Vector Laboratories, Inc., CA, USA) was added to the brains. Brains were then mounted on microscope slides and allowed $4 \mathrm{~h}$ at RT to react with DAPI before imaging on a confocal microscope. Microscope settings were kept constant across the techniques. Images of three representative brains from each technique are shown.

In each technique, Per staining is visible in the larval clock neurons, indicating the correct antibody specificity. The quantity of staining for each lobe was analyzed by examining the volume elements/ volumetric pixels (voxels) present in each image. A voxel is defined by the $3 D$ coordinate, color and intensity (of our signal being quantified) at that coordinate. Voxels above a threshold of 3000 were measured for the entirety of Per staining using the Voxel Counter algorithm of ImageJ [8]. To determine if there was a significant difference between each staining technique and the basic staining protocol, a t-test was applied. The collagenase technique showed an insignificant increase in the total Per signal. Both freezing techniques produced significant
Figure 2. Detail of third instar larval brains focusing on anti-Per immunofluorescence in axons shows quantitative and qualitative differences. Oregon- $R$ third instar larval brains were dissected and stained using the techniques indicated. Anti-Per was used to stain clock neurons. Brains were imaged and analyzed as above, focusing on the axons displayed. Maximum projections from three representative brains from each technique are shown. Scale bars: $20 \mu \mathrm{m}$. A dotted-outline on the diagram, described above, shows the location of enlargement. increases in total Per signal compared to brains treated with the basic technique (Figure 1). The freezing methods also produced more detailed staining as the axons of the clock neurons are more broadly stained (Figure 2). These data suggest that the freezing techniques allow for more uniform permeation of multiple larval brains, while still allowing for robust staining of target proteins. Phalloidin staining reveals stereotypical actin structures for basic, slow freeze, and fast freeze with no increase (compared by t-test) in phalloidin voxel counts above a threshold of 15,000 (Figure 3 ). This suggests that staining of larger neuronal structures is not significantly affected by the freezing methods. Collagenase brains had elongated morphology, and this is apparent in the phalloidin staining (Figure 3). DAPI was used to visualize the morphology of the brain (Figure 4). The overall shape 
Figure 3. Comparison of immunofluorescence techniques reveals equal staining of F-actin, but reveals distortion of lobe shape upon enzyme treatment. Oregon-R third instar larval brains were dissected and stained using the indicated techniques. Phalloidin was used to stain F-actin and visualize the cytoskeleton. Brains were imaged and analyzed as above. Maximum projections from three representative brains from each technique are shown. Scale bars: $10 \mu \mathrm{m}$.

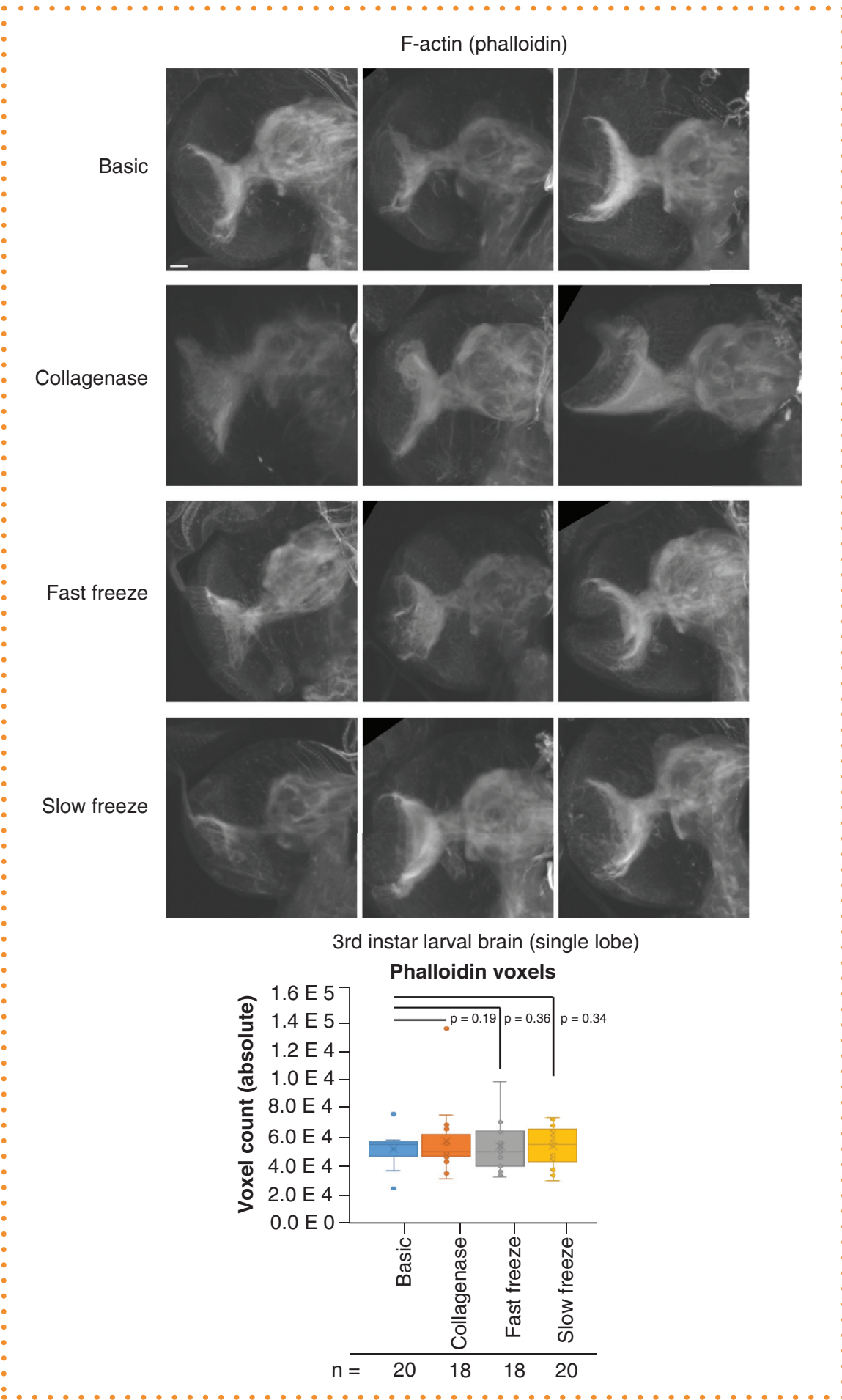

technique are best suited for maintaining brain morphology during immunofluorescence. Taken together, these results suggest that the slow and fast freezing techniques are preferable alternatives to basic and proteolytic digestion techniques for immunostaining third instar larval brains. The clock neurons and actin cytoskeleton are robustly stained in the freezing techniques, the integrity of brain morphology is uncompromised, and the results can be more reproducible, depending on the target protein.

These staining methods were additionally performed in adult brains. The collagenase technique is not shown due to the inability to produce any intact brains. Unlike larvae, there is no increase in Per 


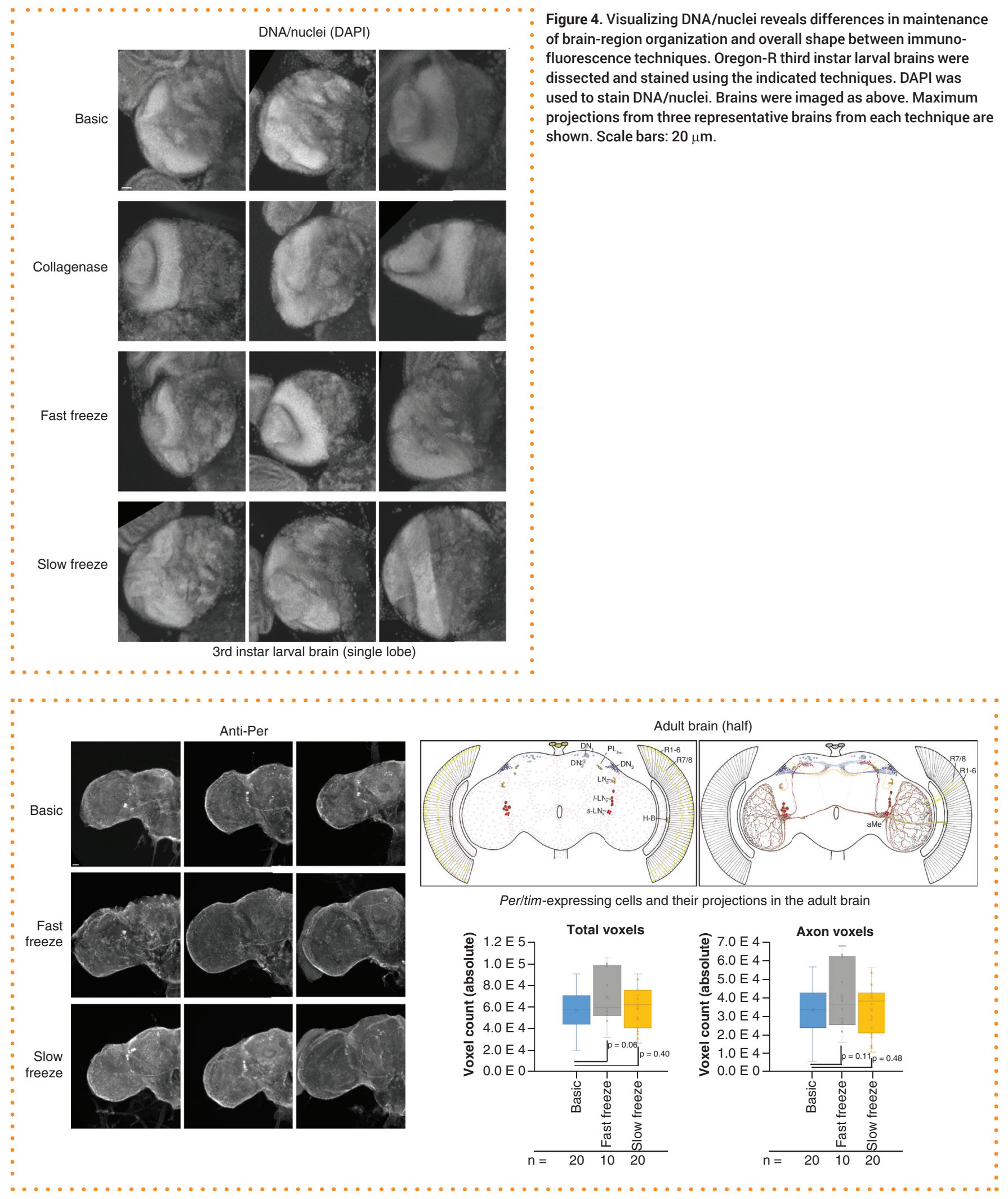

Figure 5. In adult Drosophila brains, freezing leads to qualitative but not quantitative differences in clock neuron staining. Immunostaining of Oregon- $R$ adult brains using an anti-Per antibody to reveal circadian clock neurons. Brains were imaged and analyzed as above. Maximum projections from three representative brains from each technique are shown. Scale bars: $20 \mu \mathrm{m}$. Diagrammed are the locations of per/tim-expressing cells and their projections in the adult brain, reproduced from Helfrich-Forster [9].

DN: Dorsal Neurons; H-B: Photoreceptor cells of the Hofbauer-Buchner eyelets; LN: Lateral neurons (l: large; s: small; d: dorsal); $\mathrm{PL}_{\text {tim }}$ : Posterior lateral brain cells expressing tim; R: photoreceptor nuclei (there are two groups $1-6$ and $7 / 8$ ). 
Figure 6. In adult brains, detection of F-actin is increased by including a freezing step. Oregon-R adult brains were dissected and stained using Phalloidin to stain F-actin. Brains were imaged and analyzed as above. Maximum projections from three representative brains from each technique are shown. Scale bars: $20 \mu \mathrm{m}$.
- voxel counts above a threshold of 4500 for the freezing techniques (Figure 5). In adult brains, phalloidin signal above a threshold of 6800 is increased for the two freezing techniques (Figure 6). DAPI staining reveals gaps and holes in the tissue for the fast freeze technique (Figure 7). This suggests that the optic lobes of the adult brains are negatively affected by the fast freezing technique. The reason for this is unclear,

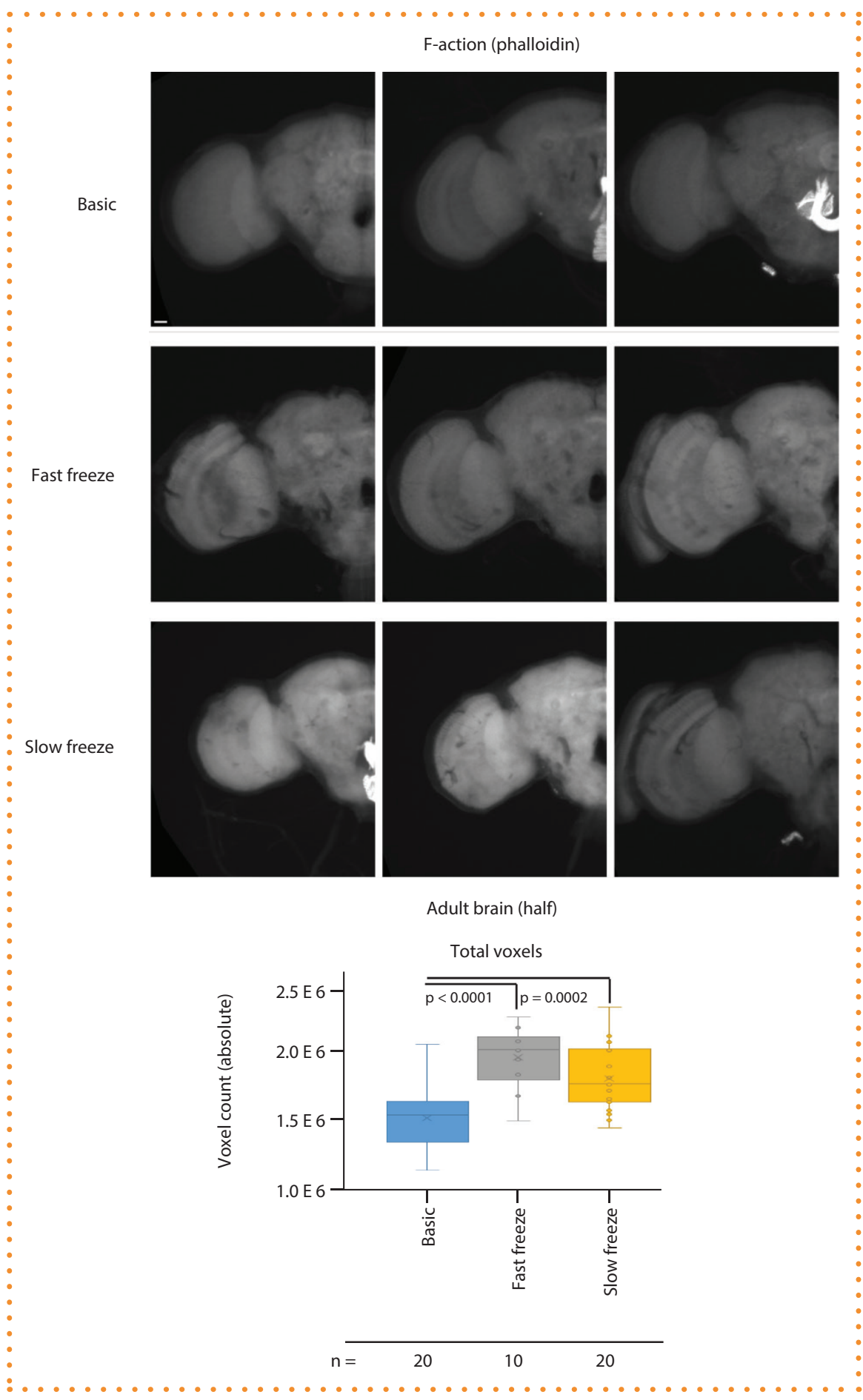

especially since the larval brains did not show the same phenomena. Overall, the adult data suggest that the slow freezing method may be a useful alternative to the basic method for adult brains.

This Benchmark introduces freezing steps in immunofluorescence techniques as alternative methods for obtaining specific antibody staining in Drosophila brains without sacrificing larval sample integrity, time, or money. In our opinion, the simple addition of a slow freezing step to immunofluorescence workflow provides increased reproducibility and appearance of immunoreactive structures without disadvantage. One possible explanation for the more uniform permeability of multiple brains is that freezing the blocking solution leads to the formation of ice crystals that penetrate the brain tissue, creating small pores that allow antibodies 
to reach their target more easily. The reason for the intact morphology of larval brains is unclear. Evidently, one freeze/ thaw cycle is enough to enhance permeability while leaving the morphology of the lobes unaltered. This may aid in studies of neurodegeneration in Drosophila that depend on understanding proper organization of neurons in both normal and disease states.

\section{ACKNOWLEDGMENTS}

We thank Jeffrey Price and Jin-Yuan Fan for the anti-Period antibody. We would also like to thank Diana Williamson for helpful input in editing the manuscript.

\section{AUTHOR CONTRIBUTIONS}

Dominic Buckley performed experiments and wrote the manuscript and protocol. Ada Thapa designed and performed experiments and contributed to writing the manuscript and protocol. Minh Nguyen designed and performed experiments. Shea M Sullivan, Vy T Ngo, Austin O Dada, and Essence Blankinship performed immunofluorescence experiments. Veronica Cloud designed experiments, performed experiments, performed data analysis, and edited and revised the cover letter, manuscript and protocol. Ryan D Mohan designed experiments, and edited and revised the cover letter, manuscript, and protocol.

\section{FINANCIAL \& COMPETING INTERESTS DISCLOSURE}

This research received funding from the University of Missouri Research Board and the NIH Academic Development Via Applied and Cutting Edge Research (ADVANCER) program. The authors have no other relevant affiliations or financial involvement with any organization or entity with a financial interest in or financial conflict with the subject matter or materials discussed in the manuscript apart from those disclosed.

No writing assistance was utilized in the production of this manuscript.

\section{OPEN ACCESS}

This work is licensed under the Attribution-NonCommercial-NoDerivatives 4.0 Unported License. To view a copy of this license, visit http://creativecommons.org/ licenses/by-nc-nd/4.0/

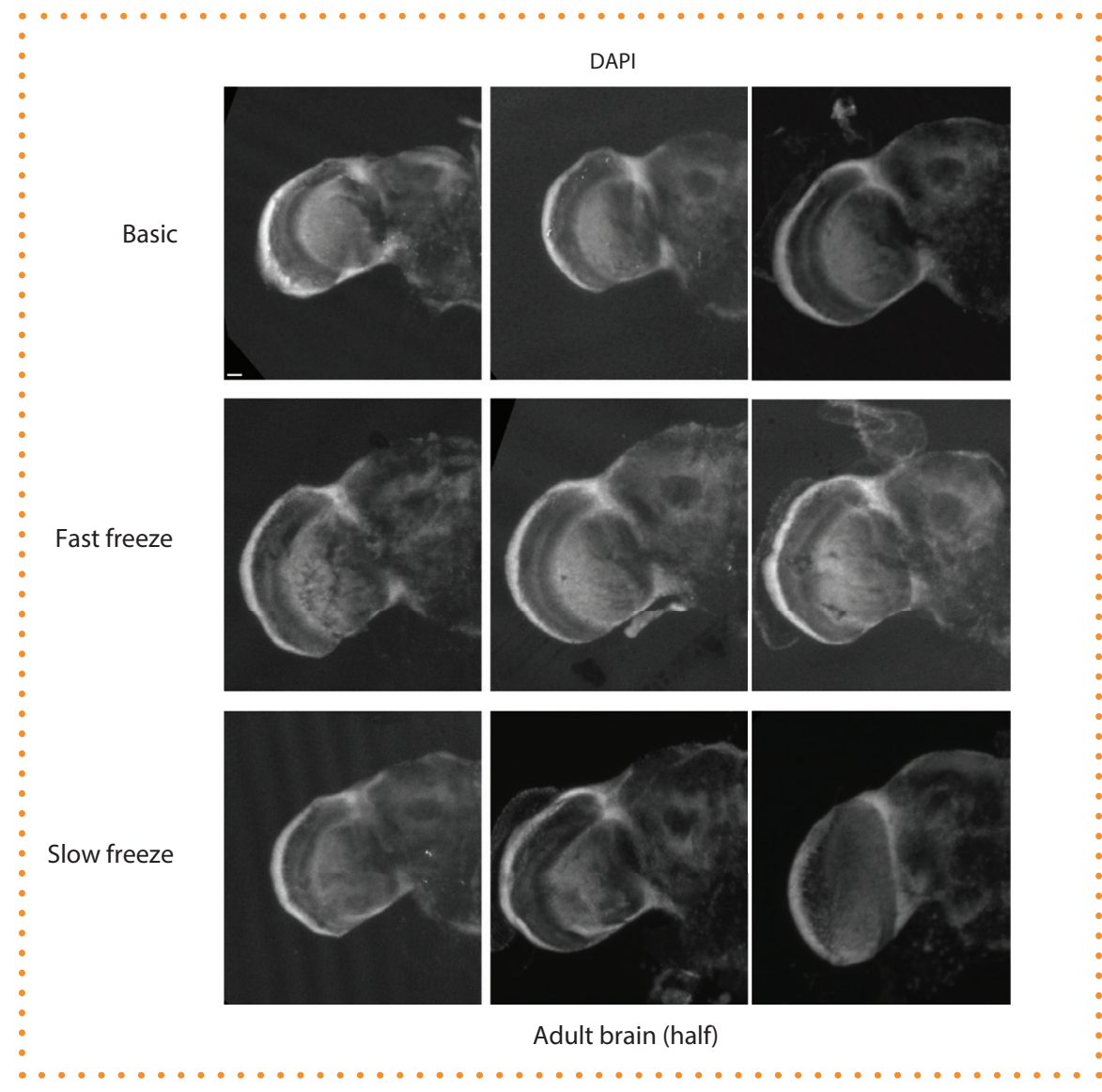

Figure 7. Imaging of DNA/nuclei in adult brains reveals comparable maintenance of overall morphology after freezing. Oregon-R adult brains were dissected and stained using each of the three techniques detailed in the protocol. DAPI was used to stain DNA/nuclei. Brains were imaged as above. Maximum projections from three representative brains from each technique are shown. Scale bars: $20 \mu \mathrm{m}$.

\section{REFERENCES}

1. Pandey UB, Nichols CD. Human disease models in Drosophila melanogaster and the role of the fly in therapeutic drug discovery. Pharmacol. Rev. 63(2), 411-436 (2011)

2. Daul AL, Komori H, Lee CY. Immunofluorescent staining of Drosophila larval brain tissue. Cold Spring Harb. Protoc. 2010(7), pdb prot5460 (2010).

3. Wu JS, Luo L. A protocol for dissecting Drosophila melanogaster brains for live imaging or immunostaining. Nat. Protoc. 1(4), 2110-2115 (2006).

4. Diaper DC, Hirth F. Immunostaining of the developing embryonic and larval Drosophila brain. Methods Mol. Biol. 1082, 3-17 (2014).

5. Kloss B, Price JL, Saez L et al. The Drosophila clock gene double-time encodes a protein closely related to human casein kinase lepsilon. Cell 94(1), 97-107 (1998).

6. Kaneko M, Helfrich-Förster C, Hall JC. Spatial and temporal expression of the period and timeless genes in the developing nervous system of Drosophila: newly identified pacemaker candidates and novel features of clock gene product cycling. J. Neurosci. 17(17), 6745-6760 (1997)

7. Muskus MJ, Preuss F, Fan J-Y, Bjes ES, Price JL. Drosophila DBT lacking protein kinase activity produces long-period and arrhythmic circadian behavioral and molecular rhythms. Mol. Cell. Biol. 27(23), 8049-8064 (2007).

8. Schindelin J, Arganda-Carreras I, Frise E et al. Fiji: an open-source platform for biological-image analysis. Nat. Methods 9(7), 676-682 (2012).

9. Helfrich-Forster $\mathrm{C}$. The neuroarchitecture of the circadian clock in the brain of Drosophila melanogaster. Microsc. Res. Techn. 62(2), 94-102 (2003). 